\title{
Estudo complementar da validade fatorial da Escala Fatorial de Satisfação em Relacionamento e predição de satisfação global com a relação
}

\author{
João Fernando Rech Wachelke - Universidade Federal de Santa Catarina \\ Alexsandro Luiz de Andrade - Universidade Federal de Santa Catarina ${ }^{1}$ \\ André Moraes Souza - Universidade Federal de Santa Catarina \\ Roberto Moraes Cruz - Universidade Federal de Santa Catarina
}

\begin{abstract}
Resumo
A pesquisa complementa a validação da Escala Fatorial de Satisfação em Relacionamento de Casal, junto a uma amostra diferenciada formada por 342 universitários em relacionamentos estáveis (52,3\% eram mulheres). Após análise fatorial foi verificada estrutura fatorial semelhante e índices de confiabilidade superiores aos do primeiro estudo, embora um dos itens tenha obtido carga inferior a 0,30 nos dois fatores. Foi realizada uma análise de regressão múltipla para relacionar as dimensões da escala com uma medida de satisfação global no relacionamento. O modelo com as duas subescalas da EFS-RC como preditores explicou 46\% da variância da satisfação com o relacionamento.
\end{abstract}

Palavras-chave: Relacionamento de casal; Satisfação no relacionamento; Relações interpessoais.

\section{Complementary factorial validity study concerning the Factorial Relationship Satisfaction Scale and prediction of global relationship satisfaction}

\begin{abstract}
The study complements the validation of the Factorial Scale of Satisfaction in Couple Relationships, within a different sample composed by 342 university students in steady relationships (52,3\% of whom were female). After a factor analysis, a similar factor structure and superior reliability indexes were found, in comparison with the first study, although one item has obtained a loading lower than 0,30 on the two factors. A multiple regression analysis was carried on to relate the scale's dimensions to a measure of global relationship satisfaction. The model including both EFS-RC sub-scales as predictors has explained $46 \%$ of relationship satisfaction variance.

Keywords: Couple relationship; Relationship satisfaction; Interpersonal relationships.
\end{abstract}

\section{Introdução}

O presente estudo apresenta dados complementares de validação da Escala Fatorial de Satisfação com o Relacionamento de Casal, a EFS-RC (Wachelke, Andrade, Cruz, Faggiani \& Natividade, 2004). Trata-se de um instrumento auto-administrado breve, formado por nove itens do tipo Likert, subdivididos em duas dimensões de avaliação de esferas específicas do relacionamento de casal: atração física e sexualidade, e afinidades de interesses e comportamentos entre companheiros de relação.

O estudo de validação da EFS-RC foi realizado em uma amostra de 364 pessoas das cidades de Florianópolis e Porto Alegre que referiam estar participando de algum tipo de relação de casal à época do inquérito, abordadas em espaços públicos urbanos. A estrutura da EFS-RC revelou-se composta por dois fatores que explicaram $51,5 \%$ da variância dos dados, correspondentes às duas dimensões mencionadas, cujos índices de confiabilidade mostraram-se aceitáveis para novas escalas, segundo critério de Nunnally (1978): a subescala SAFS, com cinco itens, (relacionada a atração física e sexualidade) teve alfa de Cronbach com o valor de 0,76 , e a subescala SAIC (afinidades de comportamentos e interesses), formada por quatro itens, obteve índice 0,61 (Wachelke e colaboradores, 2004).

Com base na literatura científica sobre $O$ fenômeno da satisfação em relacionamentos, o construto foi definido como uma avaliação individual dos benefícios originados de uma relação amorosa ou sexual em qualquer grau de intimidade (Arriaga, 2001; Rusbult, 1983; Wachelke e colaboradores, 2004). Foi considerado que os dois fatores da EFC-RC representam satisfação com aspectos delimitados do relacionamento, não cobrindo todos os campos da relação. A escala baseia-se no modelo de Fletcher, Simpson e Thomas (2000) do construto

\footnotetext{
${ }^{1}$ Endereço para correspondência:

Rua Servidão Enedina Pacheco Jacinto, 74 - Itacorubi - 88034-140 - Florianópolis-SC

Telefone: (48) 3334-3681

E-mail: alexsandro.deandrade@yahoo.com
} 
qualidade do relacionamento, formado por componentes de ordem hierárquica inferior, relativamente independentes, dentre os quais se inclui a satisfação com o relacionamento. Segundo esse modelo, a qualidade global do relacionamento de casal não fica relacionada a um componente apenas, mas sim a seis componentes singulares: satisfação, companheirismo, confiança, intimidade, paixão e amor. A satisfação com o relacionamento de casal é entendida como um componente da qualidade de relacionamento percebida. Por sua vez, conforme Wachelke e colaboradores (2004), a satisfação também seria um fator superior a outros, referentes a esferas específicas da relação de casal. É nesse contexto que podem ser enquadradas as dimensões mensuradas pela EFS-RC.

No presente estudo visou-se realizar uma replicação do estudo de validação junto a uma população relativamente diferenciada, formada, nesse caso, somente por estudantes universitários que estivessem namorando ou casados (em contraste ao primeiro estudo, em que pessoas que estavam participando de relações amorosas informais também compuseram a amostra). Nesse sentido, desejavase verificar se a estrutura fatorial e os índices de confiabilidade obtidos poderiam ser afetados pelas características demográficas da amostra. Partiu-se da hipótese de que a confiabilidade e dimensionalidade da amostra estão ligadas principalmente às características específicas dos relacionamentos, que assumem configurações variadas, independentemente de uma certa homogeneidade da população pesquisada, e, portanto, espera-se obter indicações semelhantes às do estudo de 2004.

Adicionalmente, como complemento dos resultados do processo de validação da EFS-RC, buscouse verificar quão bem as dimensões da escala poderiam prever uma satisfação global com o relacionamento de casal. Por meio desse segundo objetivo, foi estudada a importância desses aspectos para a avaliação da satisfação nas relações amorosas.

\section{Método}

\section{Participantes}

Participaram da pesquisa 342 estudantes de cursos de graduação da Universidade Federal de Santa Catarina. Desses, 179 (52,3\%) foram do sexo feminino, indicando uma amostra razoavelmente equilibrada em relação à pertença a categorias sexuais. As idades dos participantes variaram de 17 a 35 anos, com média de 21 anos e 11 meses (desvio padrão $=3$ anos e 9 meses). Todos os participantes declararam estar namorando ou casados à época do inquérito. Do total de participantes, 21,9\% participavam de relacionamentos de até 6 meses, 31,9\% referiam namoros ou casamentos com duração entre 7 meses e 2 anos, 32,7\% apontavam duração de relacionamentos de 2 a 5 anos e 13,5\%, há mais de 5 anos.

Foram contemplados na amostra estudantes de 28 cursos universitários de graduação, sendo $38,3 \%$ dos participantes estudantes do Centro de Ciências Tecnológicas (incluindo os cursos das engenharias, computação e sistemas de informação), 21,6\% do Centro de Filosofia e Ciências Humanas (psicologia, ciências sociais, filosofia e história), 19,9\% do Centro SócioEconômico (administração, direito, economia, ciências contábeis, serviço social), 8,2\% do Centro de Educação (pedagogia e biblioteconomia), 4,1\% do Centro de Comunicação e Expressão (jornalismo e letras) e os restantes 7,9\% dos demais centros (incluindo os cursos de farmácia, química, ciências biológicas, educação física e física).

\section{Instrumento}

Foi utilizado como instrumento um questionário auto-administrado, que continha itens de caracterização dos participantes (sexo, idade, duração do relacionamento e curso na universidade), a Escala Fatorial de Satisfação com Relacionamento de Casal - EFC-RC (Wachelke e colaboradores, 2004) e uma medida de satisfação global com relacionamento (Rusbult, 1983). Foi realizada uma pequena modificação no item 8 da EFS-RC. A expressão "engajar-me em situações de contato físico" foi substituída por "envolver-me em situações de contato físico", considerada semanticamente mais adequada, diminuindo a possibilidade de dificuldade de entendimento por parte dos participantes.

A respeito da satisfação global com o relacionamento amoroso, é recomendado que ela seja medida por meio de itens avaliativos amplos, não ligados a contextos específicos da relação, deixando ao sujeito a tarefa de indicar uma avaliação geral (Fincham \& Bradbury, 1987). Escolheu-se fazê-lo por meio da medida utilizada por Rusbult (1983), que possui apenas três itens ["estou satisfeito com meu relacionamento", "estou satisfeito com meu companheiro(a) no que diz respeito a seu papel no relacionamento" e "estou satisfeito com meu relacionamento com meu companheiro(a)"], mas alta confiabilidade. As duas medidas estavam no formato Likert de 5 pontos, sendo $1=$ "discordo fortemente" e $5=$ "concordo fortemente".

\section{Procedimento e análise}

Após agendamento com os professores dos cursos universitários, os questionários foram aplicados coletivamente em sala de aula, sempre por um casal de assistentes de pesquisa. Os aplicadores primeiramente perguntaram aos presentes nas aulas se eles estavam namorando ou eram casados e distribuíram os 
questionários somente às pessoas que responderam afirmativamente. Os dados foram analisados com o auxílio do programa informático Statistical Package for the Social Sciences, versão 11.

\section{Resultados}

Foi solicitada primeiramente uma análise dos componentes principais para verificar a dimensionalidade da escala. O KMO apresentou o valor de 0,78, aceitável para análise fatorial, apesar de o valor ideal ser 0,80 ou mais (Pereira, 1999). O Teste de Esfericidade de Bartlett foi significativo no nível de 0,0001\%. Como no primeiro estudo (Wachelke e colaboradores, 2004), o gráfico de sedimentação sugeriu a extração de dois fatores, que explicavam 53\% da variância. Foi realizada a análise fatorial com rotação varimax com extração de dois fatores, pedindo-se a supressão de cargas fatoriais inferiores a 0,30. Conforme é apresentado na Tabela 1, os dados repetiram a configuração fatorial com dois fatores observada no primeiro estudo (Wachelke e colaboradores, 2004), com uma exceção: um dos itens não teve carga mínima em nenhum dos dois fatores. Trata-se do item que apresentou carga mais baixa no primeiro estudo, referente à reprovação de ações do companheiro de relação.

Tabela 1 - Distribuição das cargas fatoriais e comunalidade dos itens de análise fatorial exploratória

\begin{tabular}{|c|c|c|c|}
\hline \multirow[b]{2}{*}{ Itens } & \multicolumn{2}{|c|}{ Fatores } & \multirow[b]{2}{*}{$h^{2}$} \\
\hline & SAFS & SAIC & \\
\hline 2. Considero meu companheiro(a) bonito. & 0,830 & & 0,591 \\
\hline 4. Meu companheiro(a) é fisicamente atraente para mim. & 0,742 & & 0,519 \\
\hline 7. (reverso) A aparência de meu companheiro(a) NÃO é a ideal para mim. & 0,674 & & 0,432 \\
\hline 9. As situações de contato físico entre meu companheiro(a) e eu são muito prazerosas. & 0,490 & & 0,408 \\
\hline $\begin{array}{l}\text { 8. Sinto muita vontade de envolver-me em situações de contato físico com meu } \\
\text { companheiro(a). }\end{array}$ & 0,480 & & 0,381 \\
\hline 1. Meu companheiro(a) e eu temos muitas idéias e interesses em comum. & & 0,793 & 0,412 \\
\hline 6. Meu companheiro(a) e eu gostamos de participar de atividades similares. & & 0,582 & 0,308 \\
\hline $\begin{array}{l}\text { 3. Meu companheiro(a) e eu temos interesses e expectativas compatíveis em relação a } \\
\text { nosso futuro profissional. }\end{array}$ & & 0,498 & 0,253 \\
\hline 5. (reverso) Reprovo muitas atitudes de meu companheiro(a). & & & 0,054 \\
\hline
\end{tabular}

SAFS: Satisfação com Atração Física e Sexualidade; SAIC: Satisfação com Afinidade de Interesses e Comportamentos. Método de extração: Fatoração do Eixo Principal. Método de rotação: varimax com normalização Kaiser. Obs.: cargas fatoriais com valor inferior a 0,30 foram suprimidas.

Em vista disso, o item foi excluído da escala para os fins da presente investigação. Resultou disso que o fator SAFS foi composto por cinco itens, e o fator SAIC, por três. O alfa de Cronbach calculado para o SAFS foi de 0,80 , e para o SAIC de 0,68. São valores superiores aos calculados no estudo de validação original, de 0,76 e 0,61 , respectivamente. $O$ alfa de Cronbach calculado para a medida de satisfação global foi de 0,90 .

Foi efetuada posteriormente uma análise de regressão linear múltipla (Dancey \& Reidy, 2006), com método passo a passo (Abbad \& Torres, 2002) para chegar a um modelo que permitisse prever a variável dependente satisfação com o relacionamento a partir das subescalas SAFS e SAIC (ver Tabela 2). O modelo com as duas variáveis como preditoras foi o que melhor explicou a variável dependente, com $46 \%$ da variância compartilhada com a satisfação $\left(\mathrm{R}^{2}=0,46\right)$. A variável SAIC foi a que melhor explicou a variância da satisfação global com o namoro: $37 \%$, e, portanto, o acréscimo da SAFS ao modelo acrescentou apenas $9 \%$ de variância compartilhada. No modelo 2, os coeficientes beta obtidos foram 0,47 para o SAIC (t $(339)=10,657 ; \mathrm{p}<0,001)$ e 0,33 para o SAFS ( $\mathrm{t}(339)=7,458 ; \mathrm{p}<0,001)$.

Tabela 2 - Modelos da análise de regressão múltipla para a satisfação global do relacionamento a partir das dimensões da EFS-RC

\begin{tabular}{ccccccc}
\hline Modelo & Preditores & $\mathrm{R}$ & $\mathrm{R}^{2}$ & Beta & $\mathrm{F}$ & $\mathrm{P}$ \\
\hline 1 & SAIC & 0,610 & 0,372 & 0,610 & $201,105(1,340)$ & $<0,001$ \\
2 & SAIC & 0,678 & 0,460 & 0,470 & $144,512(2,339)$ & $<0,001$ \\
& SAFS & & & 0,329 & & \\
\hline
\end{tabular}

Método: stepwise. Variável dependente: medida de satisfação global com o relacionamento. 


\section{Discussão}

Os resultados confirmam a estrutura fatorial da EFS-RC, apontando para uma relativa robustez do instrumento e dos aspectos analisados ao tratar com populações de características demográficas distintas. Ressalta-se, contudo, que um dos itens teve que ser desconsiderado na presente investigação em virtude da baixa carga num dos fatores, o que demonstra que alguns itens podem apresentar flutuações na representatividade do construto de satisfação com o relacionamento. Os índices de confiabilidade foram mais elevados no presente estudo que no realizado por Wachelke e colaboradores (2004), o que pode ser um indicativo de que a escala é mais eficiente quando os participantes estão inseridos em relações amorosas mais estáveis, visto que no primeiro estudo foi permitida a inclusão de casais que não necessitavam estar namorando ou casados.

Com os resultados da regressão múltipla tem-se mais evidências de um nexo associativo entre satisfação com aspectos do relacionamento e a satisfação global com o relacionamento de casal, em conformidade com o modelo de qualidade do relacionamento de Fletcher, Simpson \& Thomas (2000). Das duas dimensões cobertas pela EFS-RC, a satisfação com afinidade de interesses e comportamentos parece ser a mais relevante, permitindo explicar uma maior parcela da variância da satisfação geral com a relação amorosa.

O estudo fornece um complemento necessário para o emprego em pesquisas da EFS-RC. Contudo, tanto a presente investigação como o primeiro estudo de validação possuem a limitação de terem sido realizados no sul do Brasil. Além disso, embora demonstre utilidade como medida no contexto de pesquisas sobre relacionamentos de casal, a EFS-RC cobre uma parcela relativamente limitada das esferas da relação de casal. Há carência de medidas com maior cobertura do construto e qualidades psicométricas ainda melhores. Evidentemente, há abertura para outras abordagens no modo de operacionalizar o estudo da satisfação em relacionamento de casal. Como exemplo, pode-se mencionar o estudo de Norgren, Souza, Kaslow, Hammerschmidt \& Sharlin (2004), que, em pesquisa sobre satisfação conjugal em casamentos de longa duração, encontraram correlações significativas de satisfação no relacionamento com outros construtos: felicidade do casal, coesão, ajustamento conjugal, expressão de afeto, comunicação, solução de problemas e consenso. Nesse sentido, julga-se que é justificada a construção e validação de medidas mais abrangentes sobre qualidade do relacionamento e satisfação com esferas do relacionamento de casal.

\section{Referências}

Abbad, G. \& Torres, C. V. (2002). Regressão múltipla stepwise e hierárquica em psicologia organizacional: aplicações, problemas e soluções. Estudos de Psicologia (Natal), 7(especial), 19-29.

Arriaga, X. B. (2001). The ups and downs of dating: fluctuations in satisfaction in newly formed romantic relationships. Journal of Personality \& Social Psychology, 80(5), 754-765.

Dancey, C. P. \& Reidy, J. (2006). Estatística sem matemática para psicologia usando SPSS para Windows. Porto Alegre: Artes Médicas.

Fincham, F. D. \& Bradbury, T. N. (1987). The assessment of marital quality: A re-evaluation. Journal of Marriage and the Family, 49, 797-810.

Fletcher, G. J. O., Simpson, J. A. \& Thomas. G. (2000). The measurement of perceived relationship quality components: A confirmatory factor analytic approach. Personality and Social Psychology Bulletin, 26(3) 340-354.

Norgen, M. B. P., Souza, R. M., Kaslow, F., Hammerschmidt, H. \& Sharlin, S. A. (2004). Satisfação conjugal em casamentos de longa duração: uma construção possível. Estudos de Psicologia (Natal), 9(3), 575-584.

Nunnally, J. C. (1978). Psychometric theory. New York: McGraw-Hill.

Pereira, J. C. R. (1999). Análise de dados qualitativos: estratégias metodológicas para as ciências da saúde, humanas e sociais. São Paulo: EDUSP.

Rusbult, C. E. (1983). A longitudinal test of the investment model: The development (and deterioration) of satisfaction and commitment in heterosexual involvements. Journal of Personality and Social Psychology, 45, 101-117.

Wachelke, J. F. R., Andrade, A. L. de, Cruz, R. M.; Faggiani, R. B. \& Natividade, J. C. (2004) Medida da satisfação em relacionamento de casal. Psico-USF, 9(1), 11-18.

Recebido em junbo de 2006 Reformulado em abril de 2007 Aprovado em maio de 2007 
Sobre os autores:

João Fernando Rech Wachelke é psicólogo, pesquisador associado ao LACCOS - Laboratório de Psicossociologia da Comunicação e Cognição Social e mestre em Psicologia (área de concentração Processos Psicossociais, Saúde e Desenvolvimento Psicológico) pela Universidade Federal de Santa Catarina.

Alexsandro Luiz de Andrade é psicólogo e mestre em Psicologia (área de concentração Processos Psicossociais, Saúde e Desenvolvimento Psicológico) pela Universidade Federal de Santa Catarina.

André Moraes Souza é psicólogo formado pela Universidade Federal de Santa Catarina.

Roberto Moraes Cruz é psicólogo, mestre em Educação pela Universidade Federal da Bahia e doutor em Engenharia de Produção pela Universidade Federal de Santa Catarina, professor do curso de graduação e pósgraduação em Psicologia da Universidade Federal de Santa Catarina e coordenador do Laboratório de Psicologia do Trabalho e Ergonomia. 
